\title{
Does $\beta$-blocker prophylaxis improve survival after major noncardiac surgery?
}

Lindenauer PK, Pekow P, Wang K, Mamidi DK, Gutierrez B, Benjamin EM. Perioperative beta-blocker therapy and mortality after major noncardiac surgery. N Engl J Med 2005;353:349-61.

Background: The value of prophylactic $\beta$-blockade in reducing morbidity and mortality after major noncardiac surgery remains controversial, although limited evidence and several guidelines favour this approach.

Design: A U.S. database was used to retrospectively identify adult patients who underwent major noncardiac surgery. Using a propensity score that was calculated to reflect the likelihood of receiving prophylactic $\beta$-blockade therapy, $119632 \mathrm{pa}-$ tients who took $\beta$-blockers during the first 2 days of hospital admission were matched with 216290 patients who did not. In-hospital mortality was compared using logistic regression, incorporating the propensity score to adjust for potential between-group differences.

Results: Overall, $18 \%$ of patients received $\beta$-blockers, including $14 \%$ of patients at very low risk of death (Revised Cardiac Risk Index [RCRI] score of 0 ) and $44 \%$ of patients at high + risk $(\mathrm{RCRI} \geq 4)$. The relation $\exists$ between perioperative $\beta$-blockade therapy and the risk of death varied with cardiac risk. Among low-risk patients (RCRI $\leq 1$ ), therapy was associated with $\ddot{-}$ no benefit and possible harm, $§$ whereas for higher-risk patients
( $\mathrm{RCRI} \geq 2$ ), therapy reduced the risk of death, with the benefit increasing with increasing baseline risk (Table 1).

Commentary: The most important threat to the validity of this well-powered observational study is that unmeasured differences between the groups may have accounted for differences in outcome. The authors used several techniques, including propensity analysis, to adjust for factors that might independently reduce the risk of death for patients receiving $\beta$-blockers compared with control subjects. However, several database limitations leave open the possibility of residual confounding. First, the investigators did not know the date of surgery and defined perioperative use of $\beta$-blockers as prescription within an arbitrary time period during the hospital admission. Second, they lacked information regarding indications for $\beta$-blockers, which may have been withheld from more unstable patients. Finally, they lacked data regarding preoperative use of $\beta$-blockers, which raises the possibility that some patients in the control group experienced adverse consequences of withdrawal.

The observed relative risk or odds ratio for most treatments tends to be similar across varying

$\begin{aligned} & \text { Table 1: Adjusted odds ratios (ORs) for in-hospital } \\
& \text { death associated with perioperative } \boldsymbol{\beta} \text {-blocker } \\
& \text { therapy by Revised Cardiac Risk Index (RCRI) score }\end{aligned}$
\begin{tabular}{lc} 
RCRI score & OR (95\% confidence interval) \\
\hline 0 & $1.43(1.29-1.58)$ \\
1 & $1.13(0.99-1.30)$ \\
2 & $0.90(0.75-1.08)$ \\
3 & $0.71(0.56-0.91)$ \\
$\geq 4$ & $0.57(0.42-0.76)$ \\
\hline
\end{tabular}

baseline risks, whereas absolute risk reduction is generally greater for higher-risk patients. ${ }^{1}$ Studies of secondary prevention of myocardial infarction with $\beta$ blockers $^{2}$ and statins ${ }^{3}$ have demonstrated this phenomenon. However, in this study the odds ratio ranged from harmful to highly beneficial as the baseline risk increased from low to high. This unexpected finding is not easily explained, but it suggests that baseline cardiac risk modifies the effect of $\beta$-blockers. One possible explanation is that harmful effects of perioperative $\beta$-blockade outweigh any small advantages for lower-risk patients.

Practice implications: This study presents real-world observational data about the use of $\beta$ blockers to improve perioperative outcomes and provides limited additional support for their use in this setting. Because of design limitations, however, clinicians should interpret the conclusions cautiously. The results of 2 randomized controlled trials currently underway are awaited with anticipation.

Marius Terblanche

Neill K.J. Adhikari

Damon C. Scales

Department of Critical Care Sunnybrook and Women's College Health Sciences Centre;

Interdepartmental Division of

Critical Care

University of Toronto

Toronto, Ont.

\section{References}

1. McAlister F. Commentary: relative treatment effects are consistent across the spectrum of underlying risks... usually. Int 7 Epidemiol 2002;31:76-7.

2. The Beta-Blocker Pooling Project Research Group. The Beta-Blocker Pooling Project (BBPP): subgroup findings from randomized trials in post infarction patients. Eur Heart 7 1988;9:8-16.

3. Simes J, Furberg C, Braunwald E, Davis B, Ford I, Tonkin A, et al. Effects of pravastatin on mortality in patients with and without coronary heart disease across a broad range of cholesterol levels. The Prospective Pravastatin Pooling project. Eur Heart 7 2002;23:207-15. 